\title{
Harnessing magnetic-mechano actuation in regenerative medicine and tissue engineering
}

\author{
Lívia J. Santos ${ }^{1,2}$, Rui L. Reis ${ }^{1,2}$, and Manuela E. Gomes ${ }^{1,2}$ \\ ${ }^{1}$ 3B's Research Group, Avepark, Parque de Ciência e Tecnologia, Zona Industrial da Gandra, 4805-017 Barco GMR, Portugal \\ ${ }^{2}$ ICVS/3B's, PT Government Associate Laboratory, Portugal
}

\begin{abstract}
Mechanical stimulus is of upmost importance in tissues developmental and regeneration processes as well as in maintaining body homeostasis. Classical physiological reactions encompass an increase of blood vessel diameter upon exposure to high blood pressure, or the expansion of cortical bone after continuous high-impact exercise. At a cellular level, it is well established that extracellular stiffness, topography, and remote magnetic actuation are instructive mechanical signals for stem cell differentiation. Based on this, biomaterials and their properties can be designed to act as true stem cell regulators, eventually leading to important advances in conventional tissue engineering techniques. This review identifies the latest advances and tremendous potential of magnetic actuation within the scope of regenerative medicine and tissue engineering.
\end{abstract}

\section{Mechanotransduction, magnetic actuation and tissue} engineering - connecting the dots

Mechanical forces exert epigenetic control in tissue developmental, remodelling, and regeneration processes [1,2]. The first medical evidence of the role of mechanical forces in tissue remodelling was provided by the German surgeon Jullius Wolff $>100$ years ago after detailing the influence of mechanical load on bone mass and geometry in his study 'The Law of Bone Transformation' [3]. Striking evidence of the impact of mechanical loading on bone remodelling came from astronauts living under microgravity conditions. Lack of mechanical load led to detrimental effects such as a significant loss of bone mass, higher incidence of cardiovascular disease, and signs of accelerated biological ageing [4]. Increased mechanical load can, in contrast, have positive effects, as is the case for high-performance athletes such as Olympic fencers. These athletes undertake intermittent high-impact activity, leading to expansion of cortical bone, as well as increase of trabecular bone density and muscle mass [5]. Such strengthening is vital for the protection of the body from injuries [6].

Corresponding author: Gomes, M.E. (megomes@dep.uminho.pt).

Keywords: regenerative medicine; stem cells; magnetic-based cell manipulation; mechanosensing machinery; activation of mechanotransduction signalling pathways; magnetic biomaterials.

0167-7799/

(C) 2015 Elsevier Ltd. All rights reserved. http://dx.doi.org/10.1016/j.tibtech.2015.06.006
Physiological changes in response to mechanical load are initiated by a process called mechanotransduction, in which cells detect mechanical changes in their microenvironment through specialised machinery and then translate the information into an appropriate biological response. Mechanotransduction is mediated by structural proteins such as integrins and actomyosin fibres, which establish a physical connection between the cell and the microenvironment. When a mechanical stimulus is applied to the cell, the increase in tension is transmitted through the contraction of actomyosin fibres, a process mediated by the small GTPase Rho and Rho-associated protein kinase (ROCK) proteins [7].

Mechanotransduction is pivotal in tissue homeostasis; however, ageing and disease may impair this physiological process, giving rise to musculoskeletal disorders [8-14], in particular, the loss of muscle mass and function, decreased

\section{Glossary}

Actomyosin: actin and myosin fibres present in the cytoskeleton.

Emerin: a protein of the inner nuclear envelope.

Integrins: transmembrane proteins that convey forces inside and out of the cell and activate downstream signalling pathways mediated by the establishment of large integrin-scaffold protein assemblies called focal adhesions.

iPS cells: induced pluripotent stem cells obtained from adult cells, through genetic reprograming.

Magnetic actuation: technique of activating mechanotransduction signalling pathways in cells through the application of magnetic force. The established field can be static being the force constant, or oscillating showing a pulsed behaviour. Activation of mechanotransduction signalling pathways can occur within minutes. Magnetic bioreactor: magnetic array capable of delivering an oscillating magnetic field to cells growing in standard tissue culture surfaces or biomaterials. Although the frequency is tunable, values often range between 1 and $3 \mathrm{~Hz}$, which are the closest to physiological stress.

Mechanical cues: physical stimulus present in the cell microenvironment.

PEMF therapy: delivery of pulsed magnetic force for healing purposes, for example, to bridge a non- or delayed bone fracture.

Reverse piezoelectric effect: mechanical deformation caused by an electrical field. Rho-GTPases: small signalling G proteins implicated in numerous cells processes such as cytoskeleton dynamics.

Scaffold: structures from natural of synthetic origin that provides cells physical support and guidance.

SPIONs: iron oxide nanoparticles with supermagnetic properties used in stem cell guidance and magnetic resonance.

Stem cell: undifferentiated cell with the potential of generating several specialised cell lineages.

Stretch-activated ion channels: ion channels gated in response to alterations of the conformation of the cytoplasm membrane.

Tissue graft: tissue portion from autologous, allogeneic or xenogeneic source, employed to repair damaged tissue.

Tissue engineering: use of a combination of materials, cells, active molecules and engineering principles to restore the biological function of a damaged tissue or organ. 
healing capacity, [15], and osteoporosis [16,17]. The fact that osteoporosis is aggravated in menopause, during which oestrogen levels are reduced, may in part be explained by the role of oestrogen as a mechanosensitiser hormone [8]. Sustaining physiological mechanotransduction is thus crucial for maintaining tissue homeostasis and preventing musculoskeletal disorders.

An effective strategy for improving or sustaining mechanotransduction is magnetic actuation (see Glossary), which has been available for nearly three decades in the form of FDA-approved pulsed electromagnetic field (PEMF) therapy [18]. Owing to recent progress in nanomedicine and molecular biology, we can begin to understand the signalling pathways involved in mechanotransduction and exploit them with magnetic actuation for a myriad of applications: stem cell differentiation and homing to injury sites, as well as tissue engineering strategies [19-25]. In tissue engineering, magnetic actuation can allow for better seeding in 3D scaffolds [26-28], and can be used in scaffold-free approaches to build tissues bottom-up from mesenchymal stem cells (MSCs) or induced pluripotent stem cells (iPSCs) [29-31].

We discuss the fundamentals of mechanotransduction, the potential use of magnetic actuation in stem cell differentiation and in several aspects of tissue engineering, focusing on the musculoskeletal system, given its vulnerability to physical strain, and because musculoskeletal disorders are predicted to rise due to an increase of the ageing population and extension of life expectancy [32,33]. Understanding mechanotransduction and identifying the potential uses of magnetic actuation will open new and vibrant avenues for musculoskeletal tissue engineering and regenerative medicine strategies.

\section{Box 1. Overview of proteins involved in mechanotransduction}

Focal adhesion (FA) signalling, actomyosin contraction, stretchactivated ion channels, and nuclear associated proteins [26] are all important for mechanotransduction (Figure I). Of pivotal importance are integrins, transmembrane heterodimers proteins composed of $\alpha$ and $\beta$ subunits that physically couple the ECM to the cytoskeleton by linker proteins such as talin and vinculin [27]. The cytoskeleton, composed of actin and myosin fibres, physically bridges the ECM or the cell membrane to the nucleus. Although the nucleus is the largest and stiffest organelle in the cell, it is still susceptible to mechanical forces conveyed through the cytoskeleton [28]. In the nucleus, lamins, transcription regulators Yes-associated protein and tafazzin (YAP/TAZ), myocardin-related transcription factor A (MRTF-A) and nuclear factor (NF)- $\mathrm{KB}$ all participate in gene regulation triggered by mechanical cues $[29,30]$.
Integrins convey forces between inside and outside the cell and activate downstream signalling pathways. This mechanotransduction is made possible by the establishment of large integrin-scaffold protein assemblies called FAs. Besides integrins, cells can perceive external mechanical stimuli through many other proteins such as cadherins, catenins, the components of the cytoskeleton and nucleoskeleton, stretch-activated ion-channels, and growth factor receptors. Similar to integrins, cadherins are transmembrane proteins and important signalling hubs, but instead of linking the ECM to the cell, they mediate cell-cell communication. In classical cadherins, the cytoplasmic motif is linked to $\beta$-catenin. However, because $\beta$-catenin is also implicated in transcriptional processes, it may be translocated from cadherins to the nucleus in response to intracellular tension, mediated by signalling components such as the small GTPase Rho and ROCK.
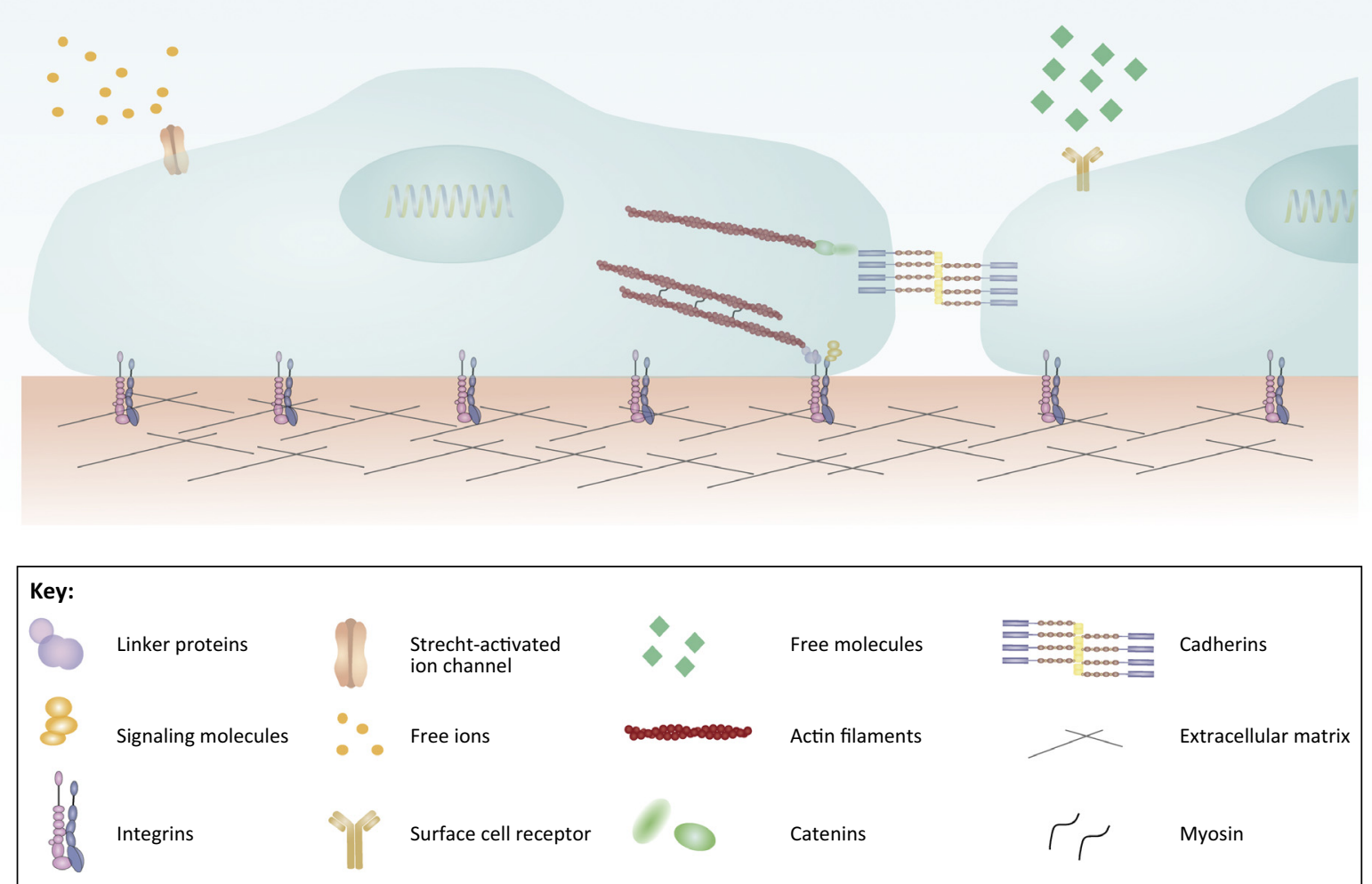

$\overline{\text { TRENDS in Biotechnology }}$ 


\section{Sensing and integrating mechanical cues in cells}

The study of mechanotransduction is a growing area of investigation and has revealed a variety of mechanosensors and signalling models (Box 1).

Extracellular stiffness is probably the best-studied mechanical property that has been investigated in the context of stem cell differentiation [34-36] (Table 1). Stem cells interpret changes in stiffness as alterations in the adhesive ligand presentation and this interpretation has a regulatory role in stem cell fate [37]. However, recent findings show that mechanotransduction is not restricted to cell surface receptors and adhesion, but can occur directly through the nucleus via phosphorylation of emerin [38]. In addition to stiffness, stem cell differentiation can be regulated by topographical cues [39-42]. Integrins also play a critical role in this process by mediating cell adhesion to micro- or nanoscale topographic features, resulting in the contraction of the cytoskeleton and activation of biochemical signalling pathways [43]. In addition to stiffness and topography, other important physical cues such as mechanical loading and shear stress can modulate mechanotransduction. Both mechanical loading and shear stress are important for maintenance of cell phenotype, as well as for directing stem cell fate towards a specific lineage [44].

Cell tension triggers important biochemical signalling cascades such as the Rho/ROCK and the mitogen-activated protein kinase (MAPK) pathways. The Rho/ROCK pathway has pleiotropic functions, comprising the regulation of cellular contraction, motility, morphology, polarity, and cell division, which are considered fundamental in stem cell specification $[7,45]$. The extracellular signal-regulated kinase (ERK) and p38 branches of the MAPK pathway are also activated upon mechanical stress and are critical regulators of stem cell fate [46-48]. Interestingly, sustained activation of this pathway is responsible for the regrowth of limbs in regenerative competent vertebrates such as salamander [49].

\section{Harnessing cell behaviour at distance}

The incorporation of mechanical cues such as stiffness and/ or topography into biomaterials design is certainly driving

\section{Table 1. Effect of mechanical cues in stem cell fate}

\begin{tabular}{l|l}
$\begin{array}{l}\text { Mechanical } \\
\text { stimulus }\end{array}$ & Cell fate \\
\hline Stiffness & $\begin{array}{l}\text { Stem cells exposed to substratum of } 40 \mathrm{kPa}, \\
\text { matching bone stiffness, undergo specification } \\
\text { towards the osteogenic lineage, whereas when } \\
\text { exposed to } 10 \mathrm{kPa} \text {, matching muscle rigidity, cells } \\
\text { commit towards the myogenic lineage. } \\
\text { Stem cells cultured in matrices of } 40 \mathrm{kPa} \text { display } \\
\text { greater abundance of tenogenic markers than } \\
\text { when exposed to } 20 \text { or } 80 \mathrm{kPa}\end{array}$ \\
\hline Topography & $\begin{array}{l}\text { Porous scaffolds are favourable to osteogenesis, } \\
\text { while aligned fibres provide an instructive niche } \\
\text { for the differentiation of tendon stem/progenitor } \\
\text { cells into tendon-like cells. }\end{array}$ \\
\hline $\begin{array}{l}\text { Shear stress, } \\
\text { tension, and } \\
\text { compression }\end{array}$ & $\begin{array}{l}\text { Nanopillars and nanoholes enhance } \\
\text { chondrogenesis and facilitates hyaline cartilage } \\
\text { formation }\end{array}$ \\
\hline $\begin{array}{l}\text { Shear stress modulates the endothelial cell } \\
\text { phomotes the osteogenesis }\end{array}$
\end{tabular}

the fields of regenerative medicine and tissue engineering forward. Nonetheless, the high cell throughput necessary to successfully implement clinical therapies is still challenging [50]. Previous methods relied on biomaterials being in direct contact with cells, but methods that utilise forces applied at a distance, such as the reverse piezoelectric effect and magnetic actuation would be a viable alternative to increase cell throughput [50]. Mechanotransduction protocols based on the reverse piezoelectric effect entail the delivery of nanosinusoidal vibrations (termed 'nanokicking') to stem cells. Conveyance of this force to cells can modulate cell adhesion and phenotype by activating mechanotransduction pathways to drive stem cell specification towards osteoblastogenesis [50]. Nanokicking may disrupt focal adhesions and interfere with signalling through G-proteins, or focal adhesion kinase (FAK) and ERK, which are implicated in the activation of the osteogenic transcription factor runt-related transcription factor (RUNX)2 [50]. Although the reverse piezoelectric effect enables high cell throughput, it is not as versatile as magnetic actuation, nor does it possess the capacity to activate specific mechanotransduction pathways.

Magnetic actuation using magnetic force alone is thought to deform the plasma membrane, thus causing changes in actomyosin tension levels and promoting cytoskeleton reorganisation. Magnetically induced cytoskeletal rearrangements can activate different mechanosensors embedded in the plasma membrane, such as stretch-activated ion channels and integrins [51], as well as signalling pathways such as the p38/MAPK pathway (Figure 1A) [52]. Such changes can also improve cell survival and viability, decrease the amount of apoptotic molecules (e.g., caspases), increase the amount of antiapoptotic molecules (e.g., bcl-2), diminish the expression of pluripotent genes [53], or accelerate osteoblast differentiation, bone regeneration, and mineralisation [52,54]. Although these cellular events are mediated by the Rho/ROCK and MAPK mechanotransduction signalling pathways, other pathways such as the AKT/PI3K pathway, which is linked to the regulation of the cell cycle and directly related to cell proliferation, can play an important role.

\section{Selectivity of magnetic-based approaches}

Utilising magnetic force with the addition of magnetic particles can allow for additional levels of control over specific mechanosensors and signalling pathways, and therefore, cell fate. Under these circumstances, magnetic actuation has two main components: the magnetic field and the responsive magnetic nanoparticle [55]. The magnetic field can be generated by permanent magnets or magnetic bioreactors that produce an oscillating pulse [55].

Remote magnetic actuation can be targeted and precise in comparison to techniques requiring direct contact. For example, exposing cells to a substrate with a certain physiological rigidity will deform the entire cell or portions of it indiscriminately, and has little to no control over specific mechanosensors and signalling pathways. In contrast, remote magnetic actuation in combination with magnetic nanoparticles can target individual cells through specific mechanosensors. Targeting is achieved by the functionalisation of a magnetic nanoparticle with ligands that bind to 


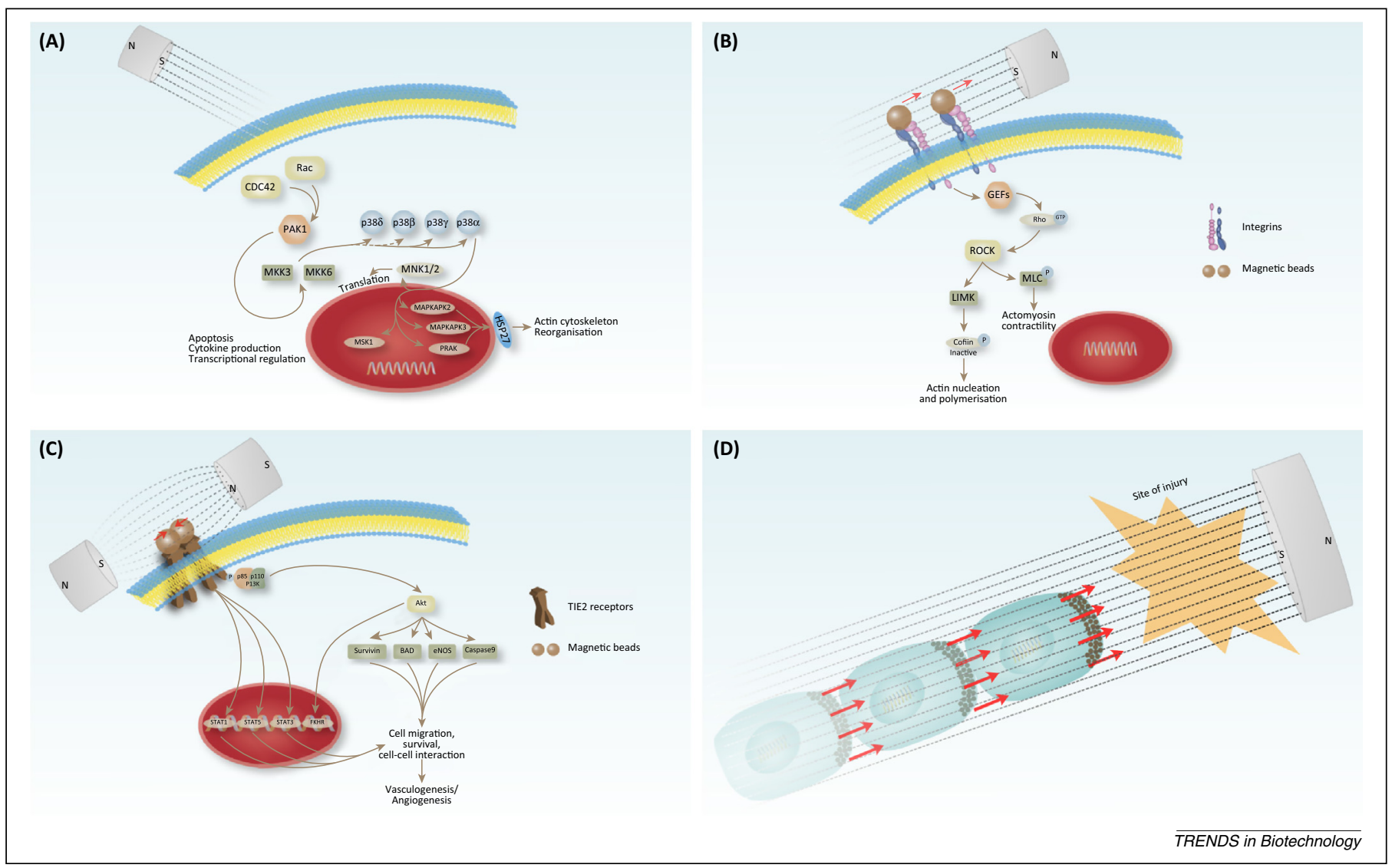

Figure 1. Magnetic actuation in cells and strategies for use in differentiation and regeneration. (A) Magnetic actuation. A magnetic field (dashed lines) directly deforms the

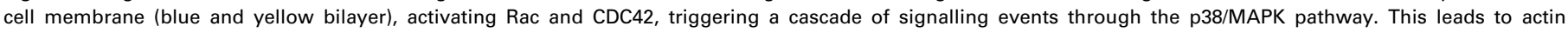
cytoskeleton reorganisation, but also other cell responses such as apoptosis, cytokine production, and transcriptional regulation. (B) Magnetic actuation with beads twisting. A magnetic field (dashed lines) torques the magnetic beads attached to integrin receptors, changing their conformation and activating cellular events such as

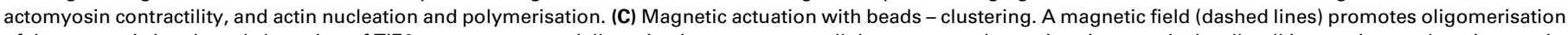
of the magnetic beads and clustering of TIE2 receptors, potentially activating numerous cellular events such as migration, survival, cell-cell interaction, and angiogenesis. (D) Cells labelled with SPIONs-labelled are steered to the site of injury through magnetic actuation. Abbreviations: BAD, BCL2-associated agonist of cell death; eNOS,

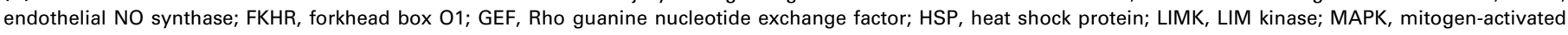
protein kinase; MKK, mitogen-activated protein kinase kinases; MLC, myosin light chain; MNK, myosin light chain kinase; PAK, p21-activated kinase; PI3K, phosphatidylinositol 3-kinase; PRAK, p38-related/activated protein kinase; Rac, ras-related C3 botulinum toxin substrate (rho family, small GTP binding protein); ROCK, Rho-associated protein kinase; SPION, superparamagnetic iron oxide nanoparticle; STAT, signal transducer and activator of transcription; TIE, tyrosine kinase with immunoglobulin and epidermal growth factor homology domains.

the desired mechanosensor. Exposure to a magnetic field will thus change the conformation of the targeted mechanosensor, either by magnetic twisting (Figure 1B) or clustering (Figure 1C), activating it and its associated downstream biochemical signalling pathways.

Magnetic particles can be functionalised with a myriad of bioactive agents, such as the integrin recognition motif arginine-glycine-aspartic acid (RGD) peptide, or antibodies to the potassium channel, subfamily $\mathrm{K}$, member 2 (TREK-1). Magnetic actuation of nanoparticles targeted to TREK-1 causes the upregulation of typical skeletal development markers, in particular the transcription factor SRY (sex determining region Y)-box 9, core binding factor $\alpha 1$ (Cbfa1) and osteopontin in vitro, and type I collagen in vivo, whereas actuation of particles targeted to RGD receptors enhance type I and II collagen synthesis both in vitro and in vivo [56].

Functionalised magnetic nanoparticles can be used in combination with osteogenic medium [57], cyclic magnetomechanical stimulation [58], and bone morphogenic protein (BMP)2 to constitute an integrated strategy to promote bone mineralisation [59].
The combination of magnetic scaffolds with magnetic force represents another valuable contribution of magnetic actuation to the field of regenerative medicine and tissue engineering. Although less selective than magnetically actuated nanoparticles, magnetically actuated scaffolds are valuable for developing proangiogenic scaffolds. The combination of both an oscillating magnetic field and a magnetic scaffold can drive organisation of endothelial cells into vessel-like structures [60]. In cardiac tissue engineering, brief stimulation of cardiac cells $(20 \mathrm{~min})$ was enough to activate the AKT pathway, known to be implicated in cell hypertrophy and survival [61].

\section{Magnetic actuation in the regeneration compartment}

The regeneration compartment or stem cell compartment encompasses the injury site where healing takes place. This is a highly complex microenvironment generally composed of stem cells, blood vessels, secreted factors, among many others such as inflammatory cells, tissue-specific cells, and extracellular matrix proteins (ECM) proteins. Endogenous tissue repair benefits from the interplay between these components with an adequate mechanical 
environment, such as stiffness, topography, and shear stress [62].

Endothelial cells in the regeneration compartment play a pivotal role in tissue regeneration and organogenesis by secreting autocrine and paracrine factors that are critical for healing, and contribute to angiogenesis by becoming the main components of the new blood vessels [63,64]. Angiogenesis can be stimulated by manipulating integrins through magnetic twisting (Figure 1B) or the endothelial receptor cell TIE Tyrosine Kinase 2 (TIE2) through magnetic clustering (Figure 1C) [65,66]. Manipulation through magnetic twisting is achieved using magnetic beads functionalised with RGD-peptide sequences to bind endothelial integrins. Application of a magnetic field perpendicular to the magnetic dipole of the bead twists the bead, thus twisting the integrin. As a result, endothelin (ET)-1 gene expression is increased by $>100 \%$ [66]. Manipulation through clustering is done using magnetic beads functionalised with TIE2 antibodies to target the receptor [65]. Application of a magnetic field clusters the targeted receptors and activates them, resulting in an increase in the tube-length of vessel-like structures [65].

Haematopoietic stem cells are important sentinels of the immune system and undertake this surveillance via clustering of $\operatorname{IgE}$ molecules bound to high-affinity $\operatorname{IgE}$ receptors, also known as FceRI. This clustering activates an intracellular calcium response by raising the intracellular calcium levels and releasing histamine [67]. Ageing has deleterious effects on haematopoietic stem cells in the stem cell compartment, thus leading to a condition called immunosenescense; a state consisting in the reduction of the competence of the immune system [67]. Decrease in immunological competence results from the progressive deterioration of innate and adaptive immune responses [68]. Magnetic actuation can be a useful tool to fight immunosenescence by mimicking the action of IgE activity. This can be achieved by using superparamagnetic nanobeads that bind to the receptor and oligomerise, clustering the FceRI receptor upon the application of a magnetic field in a similar manner as described for integrins in endothelial cells [69].

An important event in regeneration is stem cell retention and differentiation. Indeed, stem cells possess the innate ability to migrate towards the site of injury, driven by specific biochemical and mechanical cues, and to differentiate in situ to promote tissue or organ regeneration. Long-term success of certain surgical procedures still requires adjuvant therapy to enhance cell retention at a specific site. This can be achieved by labelling stem cells with superparamagnetic iron oxide nanoparticles (SPIONs) and applying a magnetic field to further guide their migration to sites requiring increased cell retention [70,71] (Figure 1D). Importantly, cell labelling with SPIONs appears not to affect viability, proliferation, or specification of MSCs into adipogenic, chondrogenic, or osteogenic lineages under in vivo conditions [72]. Similar findings were observed in magnetite-labelled neural stem cells [73].

\section{Magnetic actuation in tissue engineering}

Conventional tissue engineering strategies typically utilise a combination of cells and 3D scaffolds to regenerate or repair a portion of injured tissue. These scaffolds are vital for providing physical and mechanical support for the cells, and can also deliver chemical stimuli that boost healing such as BMP2 or basic fibroblast growth factor (bFGF) $[74,75]$. However, the regeneration process is driven not only by chemical factors, but by mechanical cues, and this is reflected by a paradigm shift in tissue engineering. Scaffold materials are now designed with specific mechanical cues such as tissue-matching stiffness or anisotropic surfaces, resulting in improved repair and regeneration.

Despite recent improvements in scaffold design, tissue engineering still faces a number of challenges. For example, once a material is implanted in the body, it may undergo significant physical and chemical changes due to protein adsorption [76] and enzymatic degradation [77], thus skewing the initially designed properties. Other limitations could be poor cell seeding and invasion due to the tortuosity and/or depth of the scaffold, or the implanted material degrading and releasing byproducts that may trigger inflammatory responses [78]. Magnetic actuation could be used to circumvent many of the current limitations of tissue engineering [79], for example, to improve cell seeding efficacies and invasion into 3D scaffolds, or as part of a scaffold-free strategy to build portions of transplantable tissues, thus bypassing the possible inflammatory response to a scaffold or its degradation byproducts. Magnetically actuated scaffolds enable mechanical stimulation of cells, but also building cell and drug delivery systems on demand. The possibility of selectively activating mechanotransduction pathways to harness stem cell fate [80,81], as well as exerting a modulatory effect of the immune system [64], makes this approach all the more attractive.

\section{Magnetic-assisted tissue engineering (Mag-TE)}

Mag-TE involves cell labelling with magnetic particles, which grants them magnetic properties (Figure 2A). In the presence of proper culturing conditions and with the application of magnetic force, it is possible to build scaffoldfree portions of skin, liver, or muscle tissue in a variety of shapes [82]. As a proof-of-concept, oriented dense muscle sheets, strings, and rings from mouse myoblasts were produced by Mag-TE [83]. Here, cells were labelled with magnetite cationic liposomes and seeded on a low-adhesive 24-well plates with a rotating magnet underneath. Cells were pulled towards the bottom of the plate by magnetic attraction and formed a dense, multilayered sheet-like construct. To produce strings that mimic muscle fibre bundles, the cells were cultured with a steady immobile magnet underneath, leading to linear cell aggregation, giving rise to a string-like $3 \mathrm{D}$ tissue construct.

One of the challenges in the construction and maintenance of myoblast sheets and strings is that these structures shrink considerably over longer cell culture periods, and thus must be stretched, aligned, and anchored to retain their shape and properties. A myoblast cell ring can be used to achieve this function [83]. To create such rings, a suspension of myoblast cells is seeded in a low adhesive cell culture plate with a polycarbonate cylinder placed in the centre. Once the cylinder is fixed, a magnet is placed underneath to accumulate the cells on the bottom of the plate. After a few days in culture, the cell layer shrinks 
(A)

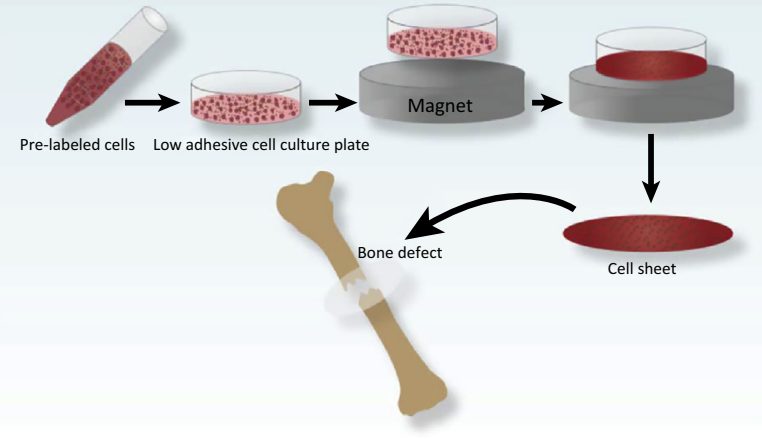

(C)

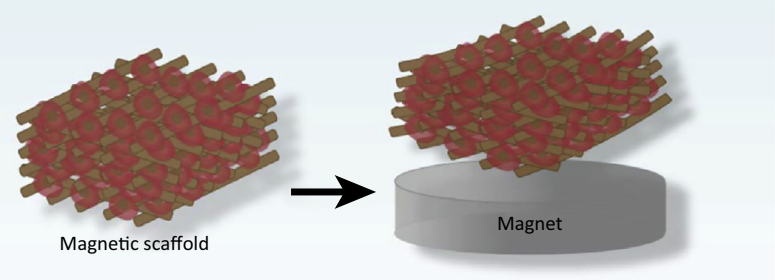

(B)

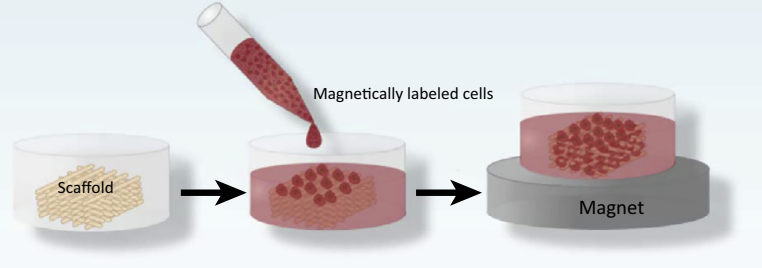

(D)

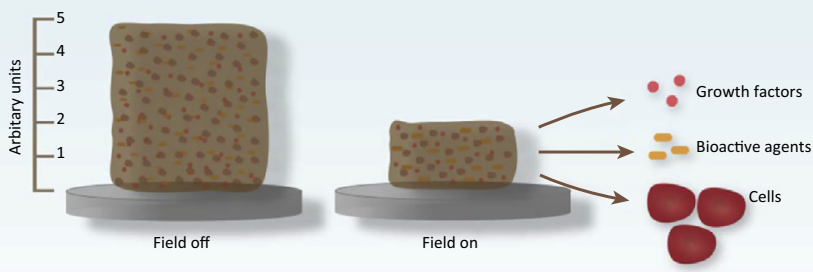

$\overline{\text { TRENDS in Biotechnology }}$

Figure 2. Magnetic actuation strategies for tissue engineering. (A) Production of a cell sheet with magnetically prelabelled cells. Cell expansion of magnetically labelled

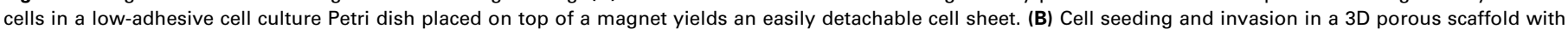
magnetically prelabelled cells. Placing a magnet underneath the tissue-engineered construct promotes cell migration into the porous scaffold and homogeneous

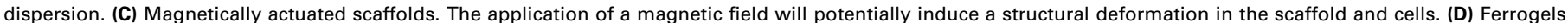
releasing cells and biomolecules on demand through the establishment of an alternating magnetic field.

and the obtained ring can be removed to be used to anchor cell sheets and strings. In addition to such proof-of-concept studies, Mag-TE has also been assessed in vivo in a bone regeneration model [29]. In this model, human multilayered MSC sheets were produced and implanted in the cranial defect of a nude rat with histological findings showing significant bone growth 14 days post-implantation. This technique has been also used in proangiogenic therapy, where multilayered adipose-derived stem cell sheets and iPSC sheets were generated and engrafted successfully in ischaemic mouse models to promote vascularisation of the hypoxic tissue [30,31].

\section{Magnetic-assisted cell seeding (Mag-seeding)}

One of the classical techniques in tissue engineering involves cell seeding into a porous $3 \mathrm{D}$ scaffold, followed by proliferation and differentiation. After acquisition of tissue-like characteristics, where cells are organised in a way resembling the native tissue and displaying markers of it (e.g., osteocalcin and alkaline phosphatase are typical of bone tissue), the construct is implanted at the injury site. Cell seeding and invasion can still be challenging due to the depth and/or tortuosity of 3D scaffolds.

Mag-seeding can overcome this limitation. In this technique, cells are labelled with magnetic particles, placed on top of the scaffold, and then driven to invade the scaffold by magnetic forces from below (Figure 2B). Mag-seeding has proven effective in promoting infiltration and distribution of magnetically labelled fibroblasts in poly(lactic-coglycolic acid) (PLGA), collagen, and polystyrene materials [26-28]. This strategy can be used with great precision, enabling distribution of cells with a high degree of spatial resolution. In this respect, magnetic tweezers are successfully used to probe and position labelled cells, allowing the seeding cells at high densities, as well as finely-tuned spatial organisation inside the construct [84].

\section{Magnetic biomaterials}

Soft and hard materials are susceptible to acquiring magnetic properties through the incorporation of magnetic iron oxide particles, as demonstrated in the development of magnetic hydrogels [85], magnetic bioactive glasses [86] magnetic blends of poly(caprolactone) (PCL)/hydroxyapatite [86], magnetic nanofibrous hydroxyapatite poly-lactide acid (PLA) [87], and magnetic PCL [88]. The methods for incorporation of magnetic particles in biomaterials include doping, blending, in situ precipitation, and the 'graftingonto' method [89]. For the purposes of tissue engineering, a material is considered to have satisfactory magnetic properties if magnetic saturation values are above $0.049 \mathrm{emu} / \mathrm{g}$ [90].

Magnetic biomaterials in combination with magnetic force provide numerous benefits unavailable to other methods and materials [55]. This approach permits the construction of magnetically actuated scaffolds with angiogenic [60] and osteogenic [88] potential, both of which are 
important for bone regeneration. Although the biophysical and biochemical mechanisms underlying this response remain elusive, it is postulated that magnetisation induces local deformation of the scaffold, which in turns mechanically stimulates cells [61] (Figure 2C).

Magnetically actuated scaffolds also provide a platform for cell and drug delivery on demand [85]. Typically, this is performed with ferromagnetic hydrogels (ferrogels), consisting of hydrogels incorporating ferromagnetic particles and further enriched with growth factors and/or cells (Figure 2D). Upon the application of a magnetic field, the hydrogel contracts, expelling the biomolecules and/or cells contained within it (Figure 2D) [85]. If the ferrogel is placed at an injury site, it can act as a potent delivery vehicle. Alternatively to release on-demand, growth factors can be covalently conjugated to the ferromagnetic particles to be delivered in a sustained manner [91]. For example, bFGF-conjugated particles embedded in fibrin hydrogels were found to encourage the proliferation and differentiation of cells to be used in the regeneration of spinal cord injuries, as compared to hydrogels containing free bFGF [91].

Magnetic materials are often obtained through the incorporation with iron oxide nanoparticles and although iron is an essential mineral of our body, the size of such particles raises a number of safety concerns with respect to their use in medicine, such as cytotoxicity, bioaccumulation, and inflammation [92]. However, such concerns have been dissipated by a number of in vivo tests demonstrating that magnetic nanofibrous scaffolds of PCL implanted subcutaneously [93] and magnetic hydroxyapatite/collagen scaffolds implanted in rabbit distal femoral epiphysis and tibial mid-diaphysis did not appear to cause inflammation [94]. Furthermore, magnetic hydroxyapatite, prepared with different ratios of magnetic particles, when implanted in rabbit bone critical size defects produced in the condyle region, had similar biocompatibility to hydroxyapatite alone [95]. Indeed, it has been observed that granting magnetic properties to biomaterials can lead to a significant increase in cell proliferation $[95,96]$. The mechanisms underlying this stimulation still need to be elucidated.

These results, as well as the fact that uptake of SPIONs has no impact on viability or differentiation potential in a diverse array of cells $[72,73]$, are good indicators of the safety of magnetically actuated materials. However, longterm in vivo tests must be undertaken before drawing further conclusions.

\section{Concluding remarks and future perspectives}

Magnetic actuation, especially in combination with magnetic nanoparticles, is a valuable tool for harnessing stem cell guidance, retention, and differentiation. This technology is also promising in promoting the occurrence of key cellular events within the regeneration compartment, such as angiogenesis, and in ameliorating competence of the immune system.

Other important contributions of magnetic actuation can be found in tissue engineering, where this technology is being effectively used in building scaffold-free tissue constructs, enhancing cell seeding efficacies in 3D scaffolds, and interestingly in magnetic responsive materials.

\section{Box 2. Outstanding questions}

- Given the complexity of the translational process from the bench to the bedside, how long will we have to wait to benefit from patient tailored mechanotherapies?

- Can we build standardised stem cell differentiation protocols based on magnetic actuation?

- What is the overall impact of magnetic actuation within the regeneration compartment?

- Functional multilayered iPSCs sheets were constructed for angiogenic therapy: what other development can we expect?

- Can selective mechanotransduction strategies based on magnetic twisting and clustering help implementing and expanding the use of MSCs and iPSCs in tissue engineering and regenerative medicine?

Indeed, another approach we strongly consider for the future lies in magnetically actuated scaffolds. These could be prevascularised and implanted, for example, in a bone defect and further exposed to a magnetic field to mechanically stimulate cells within the regeneration compartment. To conclude, we anticipate that magnetically based approaches will bring remarkable progress to regenerative medicine and will help improve the treatment efficacy of musculoskeletal disorders and fight healing constraints (Box 2).

\section{Acknowledgements}

We thank Mr Tamagno Pesqueira for his support in preparation of the Figures of this paper. This work was supported by the European project Polaris, FP7 REGPOT.

\section{References}

1 Ingber, D.E. (2006) Cellular mechanotransduction: putting all the pieces together again. FASEB J. 20, 811-827

2 Orr, A.W. et al. (2006) Mechanisms of mechanotransduction. Dev. Cell $10,11-20$

3 Brand, R.A. (2010) Biographical sketch Julius Wolff, 1836-1902. Clin. Orthop. Relat. Res. 468, 1047-1049

4 Versari, S. et al. (2013) The challenging environment on board the International Space Station affects endothelial cell function by triggering oxidative stress through thioredoxin interacting protein overexpression: the ESA-SPHINX experiment. FASEB J. 27, 4466-4475

5 Chang, G. et al. (2009) Olympic fencers: adaptations in cortical and trabecular bone determined by quantitative computed tomography. Osteoporos. Int. 20, 779-785

6 Ralphs, J.R. et al. (2002) Actin stress fibres and cell-cell adhesion molecules in tendons: organisation in vivo and response to mechanical loading of tendon cells in vitro. Matrix Biol. 21, 67-74

7 Samuel, M.S. et al. (2011) Actomyosin-mediated cellular tension drives increased tissue stiffness and beta-catenin activation to induce epidermal hyperplasia and tumor growth. Cancer Cell 19, 776-791

8 Chen, C.S. and Ingber, D.E. (1999) Tensegrity and mechanoregulation: from skeleton to cytoskeleton. Osteoarthritis Cartilage 7, 81-94

9 Ingber, D.E. (2008) Tensegrity-based mechanosensing from macro to micro. Prog. Biophys. Mol. Biol. 97, 163-179

10 Ingber, D.E. (2003) Tensegrity II. How structural networks influence cellular information processing networks. J. Cell Sci. 116, 1397-1408

11 Ingber, D.E. (1997) Tensegrity: the architectural basis of cellular mechanotransduction. Annu. Rev. Physiol. 59, 575-599

12 Ingber, D.E. et al. (2014) Tensegrity, cellular biophysics, and the mechanics of living systems. Rep. Prog. Phys. 77, 046603

13 Stamenovic, D. and Ingber, D.E. (2009) Tensegrity-guided self assembly: from molecules to living cells. Soft Matter 5, 1137-1145

14 Wang, N. et al. (2001) Mechanical behavior in living cells consistent with the tensegrity model. Proc. Natl. Acad. Sci. U.S.A. 98, 7765-7770

15 Silver, F.H. et al. (2003) Invited review: role of mechanophysiology in aging of ECM: effects of changes in mechanochemical transduction. J. Appl. Physiol (1985) 95, 2134-2141

$16 \mathrm{Wu}, \mathrm{M} . Z$. et al. (2011) Effect of aging on cellular mechanotransduction. Ageing Res. Rev. 10, 1-15 
17 Seefried, L. et al. (2010) Mechanotransduction in aging and osteoporosis. Osteologie 19, 232-239

18 Nelson, F.R.T. et al. (2003) Use of physical forces in bone healing. J. Am. Acad. Orthop. Surg. 11, 344-354

19 Tsai, M.T. et al. (2007) Pulsed electromagnetic fields affect osteoblast proliferation and differentiation in bone tissue engineering. Bioelectromagnetics 28, 519-528

20 Midura, R.J. et al. (2005) Pulsed electromagnetic field treatments enhance the healing of fibular osteotomies. J. Orthop. Res. 23, $1035-1046$

21 Adravanti, P. et al. (2014) Effect of pulsed electromagnetic field therapy in patients undergoing total knee arthroplasty: a randomised controlled trial. Int. Orthop. 38, 397-403

22 Niezgoda, J.A. et al. (2014) The management of intractable pain with adjuvant pulsed electromagnetic field therapy. Adv. Skin Wound Care $27,205-209$

23 Iannitti, T. et al. (2013) Pulsed electromagnetic field therapy for management of osteoarthritis-related pain, stiffness and physical function: clinical experience in the elderly. Clin. Interv. Aging 8, $1289-1293$

24 Hao, C.N. et al. (2014) Pulsed electromagnetic field improves cardiac function in response to myocardial infarction. Am. J. Transl. Res. 6 , 281-290

25 Ross, C.L. and Harrison, B.S. (2013) The use of magnetic field for the reduction of inflammation: a review of the history and therapeutic results. Altern. Ther. Health Med. 19, 47-54

26 Thevenot, P. et al. (2008) Magnetic nanoparticles to enhance cell seeding and distribution in tissue engineering scaffolds. Proc. IEEE Conf. Nanotechnol. 2008, 646-649

27 Shimizu, K. et al. (2006) Enhanced cell-seeding into 3D porous scaffolds by use of magnetite nanoparticles. J. Biomed. Mater. Res. B: Appl. Biomater. 77B, 265-272

28 Sasaki, T. et al. (2008) Magnetic nanoparticles for improving cell invasion in tissue engineering. J. Biomed. Mater. Res. A 86A, 969-978

29 Shimizu, K. et al. (2007) Bone tissue engineering with human mesenchymal stem cell sheets constructed using magnetite nanoparticles and magnetic force. J. Biomed. Mater. Res. B: Appl. Biomater. 82B, 471-480

30 Ishii, M. et al. (2014) Multilayered adipose-derived regenerative cell sheets created by a novel magnetite tissue engineering method for myocardial infarction. Int. J. Cardiol. 175, 545-553

31 Kito, T. et al. (2013) iPS cell sheets created by a novel magnetite tissue engineering method for reparative angiogenesis. Sci. Rep. 3, 1418

32 Kinge, J.M. et al. (2015) Musculoskeletal disorders in Norway: prevalence of chronicity and use of primary and specialist health care services. BMC Musculoskelet. Disord. 16, 1-10

33 Woolf, A.D. and Pfleger, B. (2003) Burden of major musculoskeletal conditions. Bull. World Health Organ. 81, 646-656

34 Engler, A.J. et al. (2006) Matrix elasticity directs stem cell lineage specification. Cell 126, 677-689

35 Sharma, R.I. and Snedeker, J.G. (2010) Biochemical and biomechanical gradients for directed bone marrow stromal cell differentiation toward tendon and bone. Biomaterials 31, 7695-7704

36 Chen, J. et al. (2014) Interplay of matrix stiffness and protein tethering in stem cell differentiation. Nat. Mater. 13, 979-987

37 Huebsch, N. et al. (2010) Harnessing cellular manipulation of the cellmatrix interface to control stem cell fate. Abstr. Papers Am. Chem. Soc. $239,518-526$

38 Guilluy, C. et al. (2014) Isolated nuclei adapt to force and reveal a mechanotransduction pathway in the nucleus. Nat. Cell Biol. 16, 376-381

39 Xia, L.G. et al. (2014) Effect of nano-structured bioceramic surface on osteogenic differentiation of adipose derived stem cells. Biomaterials $35,8514-8527$

40 Cheng, X.G. et al. (2014) Platelet-derived growth-factor-releasing aligned collagen-nanoparticle fibers promote the proliferation and tenogenic differentiation of adipose-derived stem cells. Acta Biomater. 10, 1360-1369

41 Kishore, V. et al. (2012) Tenogenic differentiation of human MSCs induced by the topography of electrochemically aligned collagen threads. Biomaterials 33, 2137-2144

42 Yin, Z. et al. (2010) The regulation of tendon stem cell differentiation by the alignment of nanofibers. Biomaterials 31, 2163-2175
43 Dalby, M.J. et al. (2014) Harnessing nanotopography and integrinmatrix interactions to influence stem cell fate. Nat. Mater. 13, 558-569

44 Spanoudes, K. et al. (2014) The byophysical, biochemical, and biological toobox for tenogenic phenotype mantaince in vitro. Trends Biotechnol. $32,474-482$

45 Shih, Y-R.V. et al. (2011) Matrix stiffness regulation of integrinmediated mechanotransduction during osteogenic differentiation of human mesenchymal. J. Bone Miner. Res. 26, 730-738

46 Whitmarsh, A.J. (2010) A central role for p38 MAPK in the early transcriptional response to stress. BMC Biol. 8, 1-3

47 Brien, P. et al. (2013) p38a MAPK regulates adult muscle stem cell fate by restricting progenitor proliferation during postnatal growth and repair. Stem Cells 31, 1597-1610

48 Young, J.L. et al. (2014) Mechanosensitive kinases regulate stiffnessinduced cardiomyocyte maturation. Sci. Rep. 4, 1-11

49 Yun, M.H. et al. (2014) Sustained ERK activation underlies reprogramming in regeneration-competent salamander cells and distinguishes them from their mammalian counterparts. Stem Cell Rep. 3, 15-23

50 Nikukar, H. et al. (2013) Osteogenesis of mesenchymal stem cells by nanoscale mechanotransduction. ACS Nano 7, 2758-2767

51 Sniadecki, N.J. (2010) Minireview: a tiny touch: activation of cell signaling pathways with magnetic nanoparticles. Endocrinology 151, 451-457

52 Yuge, L. et al. (2003) Physical stress by magnetic force accelerates differentiation of human osteoblasts. Biochem. Biophys. Res. Commun. $311,32-38$

53 Kurian, M.V. et al. (2012) Enhanced cell survival and diminished apoptotic response to simulated ischemia-reperfusion in H9c2 cells by magnetic field preconditioning. Apoptosis 17, 1182-1196

54 Jansen, J.H.W. et al. (2010) Stimulation of osteogenic differentiation in human osteoprogenitor cells by pulsed electromagnetic fields: an in vitro study. BMC Musculoskelet. Disord. 11, 188

55 Sensenig, R. et al. (2012) Magnetic nanoparticle-based approaches to locally target therapy and enhance tissue regeneration in vivo. Nanomedicine 7, 1425-1442

56 Kanczler, J.M. et al. (2010) Controlled differentiation of human bone marrow stromal cells using magnetic nanoparticle technology. Tissue Eng. A 16, 3241-3250

$57 \mathrm{Hu}$, B. et al. (2013) Receptor-targeted, magneto-mechanical stimulation of osteogenic differentiation of human bone marrowderived mesenchymal stem cells. Int. J. Mol. Sci. 14, 19276-19293

$58 \mathrm{Hu}$, B. et al. (2014) Control of smooth muscle alpha-actin (SMA) upregulation in HBMSCs using remote magnetic particle mechanoactivation. Nanomed. Nanotechnol. Biol. Med. 10, 45-55

59 Henstock, J.R. et al. (2014) Remotely activated mechanotransduction via magnetic nanoparticles promotes mineralization synergistically with bone morphogenetic protein 2: applications for injectable cell therapy. Stem Cells Transl. Med. 3, 1363-1374

60 Sapir, Y. et al. (2012) The promotion of in vitro vessel-like organization of endothelial cells in magnetically responsive anginate scaffolds. Biomaterials 33, 4100-4109

61 Sapir, Y. et al. (2014) Cardiac tissue engineering in magnetically actuated scaffolds. Nanotechnology 25, 1-10

62 Lane, S.W. et al. (2014) Modulating the stem cell niche for tissue regeneration. Nat. Biotechnol. 32, 795-803

63 Ding, B.S. et al. (2010) Inductive angiocrine signals from sinusoidal endothelium are required for liver regeneration. Nature 468, 310-315

64 Ding, B.S. et al. (2011) Endothelial-derived angiocrine signals induce and sustain regenerative lung alveolarization. Cell 147, 539-553

65 Lee, J.H. et al. (2010) Artificial control of cell signaling and growth by magnetic nanoparticles. Angew. Chem. Int. Ed. Engl. 49, 5698-5702

66 Chen, J. et al. (2001) Twisting integrin receptors increases endothelin-1 gene expression in endothelial cells. Am. J. Physiol. Cell Physiol. 280, C1475-C1484

67 Geiger, H. et al. (2013) The ageing haematopoietic stem cell compartment. Nat. Rev. Immunol. 13, 376-389

68 Goronzy, J.J. and Weyand, C.M. (2013) Understanding immunosenescence to improve responses to vaccines. Nat. Immunol. $14,428-436$

69 Mannix, R.J. et al. (2008) Nanomagnetic actuation of receptormediated signal transduction. Nat. Nanotechnol. 3, 36-40 
70 Chen, C.C. et al. (2013) Simple SPION incubation as an efficient intracellular labeling method for tracking neural progenitor cells using MRI. PLoS ONE 8, e56125

71 Riegler, J. et al. (2013) Superparamagnetic iron oxide nanoparticle targeting of MSCs in vascular injury. Biomaterials 34, 1987-1994

72 Yang, G. et al. (2013) Enhancement of tenogenic differentiation of human adipose stem cells by tendon-derived extracellular matrix. Biomaterials 34, 9295-9306

73 Adams, C.F. et al. (2015) Increasing magnetite contents of polymeric magnetic particles dramatically improves labeling of neural stem cell transplant populations. Nanomedicine 11, 19-29

74 Shen, J. et al. (2014) Tissue engineering bone using autologous progenitor cells in the peritoneum. PLoS ONE 9, e93514

75 Sahoo, S. et al. (2010) A bFGF-releasing silk/PLGA-based biohybrid scaffold for ligament/tendon tissue engineering using mesenchymal progenitor cells. Biomaterials 31, 2990-2998

76 Thevenot, P. et al. (2008) Surface chemistry influences implant biocompatibility. Curr. Top. Med. Chem. 8, 270-280

77 Azevedo, H.S. et al. (2003) In vitro assessment of the enzymatic degradation of several starch based biomaterials. Biomacromolecules 4, 1703-1712

78 Lee, E.A. et al. (2014) Application of magnetic nanoparticle for controlled tissue assembly and tissue engineering. Arch. Pharm. Res. $37,120-128$

79 Santo, V.E. et al. (2013) Contributions and future perspectives on the use of magnetic nanoparticles as diagnostic and therapeutic tools in the field of regenerative medicine. Expert Rev. Mol. Diagn. 13, 553-566

80 Hughes, S. et al. (2008) Selective activation of mechanosensitive ion channels using magnetic particles. J. R. Soc. Interface 5, 855-863

81 Hughes, S. et al. (2005) Magnetic micro- and nanoparticle mediated activation of mechanosensitive ion channels. Med. Eng. Phys. 27, 754-762

82 Ito, A. and Kamihira, M. (2011) Tissue engineering using magnetite nanoparticles. In Progress in Molecular Biology and Translational Science Nanoparticles in Translational Science and Medicine (Volume 104) (Antonio, V., ed.), pp. 355-395, Academic Press

83 Yamamoto, Y. et al. (2009) Preparation of artificial skeletal muscle tissues by a magnetic force-based tissue engineering technique. J. Biosci. Bioeng. 108, 538-543
84 Robert, D. et al. (2010) Magnetic micro-manipulations to probe the local physical properties of porous scaffolds and to confine stem cells. Biomaterials 31, 1586-1595

85 Cezar, C.A. et al. (2014) Biphasic ferrogels for triggered drug and cell delivery. Adv. Healthc. Mater. 3, 1869-1876

86 Wang, D. et al. (2013) One-pot synthesis of magnetic, macro/ mesoporous bioactive glasses for bone tissue engineering. Sci. Technol. Adv. Mater. 14, 1-9

87 Gloria, A. et al. (2013) Magnetic poly(epsilon-caprolactone)/iron-doped hydroxyapatite nanocomposite substrates for advanced bone tissue engineering. J. R. Soc. Interface 10, 20120833

$88 \mathrm{Kim}$, J.J. et al. (2014) Magnetic scaffolds of polycaprolactone with functionalized magnetite nanoparticles: physicochemical, mechanical, and biological properties effective for bone regeneration. RSC Adv. 4, 17325-17336

89 Li, Y.H. et al. (2013) Magnetic hydrogels and their potential biomedical applications. Adv. Funct. Mater. 23, 660-672

90 Meng, J. et al. (2013) Super-paramagnetic responsive nanofibrous scaffolds under static magnetic field enhance osteogenesis for bone repair in vivo. Sci. Rep. 3, 2655

91 Ziv-Polat, O. et al. (2012) Novel magnetic fibrin hydrogel scaffolds containing thrombin and growth factors conjugated iron oxide nanoparticles for tissue engineering. Int. J. Nanomed. 7, 1259-1274

92 Corchero, J.L. and Villaverde, A. (2009) Biomedical applications of distally controlled magnetic nanoparticles. Trends Biotechnol. 27, 468476

93 Singh, R.K. et al. (2014) Potential of magnetic nanofiber scaffolds with mechanical and biological properties applicable for bone regeneration. PLoS ONE 9, 1-16

94 Panseri, S. et al. (2012) Innovative magnetic scaffolds for orthopedic tissue engineering. J. Biomed. Mater. Res. A 100, 2278-2286

95 Panseri, S. et al. (2012) Magnetic hydroxyapatite bone substitutes to enhance tissue regeneration: evaluation in vitro using osteoblast-like cells and in vivo in a bone defect. PLoS ONE 7, e38710

96 De Santis, R. et al. (2015) Towards the design of 3D fiber-deposited poly(epsilon-caprolactone)/iron-doped hydroxyapatite nanocomposite magnetic scaffolds for bone regeneration. J. Biomed. Nanotechnol. $11,1236-1246$ 